\title{
-NOTES-
}

\section{ON DIAKOPTICS IN A CAUER-ROUTH SETTING*}

BY W. H. INGRAM (150 Claremont Ave., New York, NY 10027)

Introduction. In a digressive paragraph, $\uparrow 280$ of Vol. 1, of his famous treatise Electricity and magnetism, Maxwell defined the elements of a matrix $\boldsymbol{Y}$ of admittances such that $\boldsymbol{Y} v$ is a vector $l$ of elements having the dimension of currents and in number $v$, the number of nodes of the network. In an earlier note [2], this current was thought of as a disturbance in the equilibrium of a Kirchhoff network:

$$
\left(\begin{array}{cc}
Z & \Pi_{T} \\
\Pi & 0
\end{array}\right)\left(\begin{array}{l}
i \\
v
\end{array}\right)=\left(\begin{array}{c}
e \\
-\imath
\end{array}\right)
$$

In the present note, Maxwell's virtual currents are realized as tieline currents between co-grounded networks. The tielines constitute a dangling cutset ${ }^{1}$ and not a conventional cutset, but I deem G. Kron's term diakoptics to be descriptively appropriate to the theory.

In the case of an impedance matrix defined in situ, a network is governed by a Cauer-Routh equation of the form

$$
\left(\begin{array}{cc}
Z & \Pi_{T} \\
\Pi & 0
\end{array}\right)\left(\begin{array}{l}
i \\
v
\end{array}\right)=\left(\begin{array}{l}
e \\
0
\end{array}\right)
$$

[1, Eq. (4)]. The graph may be found to have lines or surfaces of cleavage through the grounding point which separate the network into connected (i.e., self-connected) inductively isolated co-grounded subnetworks interconnected by tielines, as is represented in the simple case of Fig. 1. For this separation to have computational advantage it is sufficient, by an ordered indexing of branches and nodes, to particularize the branchimpedance and node-incidence matrices as

$$
\begin{aligned}
& \left(\begin{array}{ll}
Z & 0 \\
0 & z
\end{array}\right)=\left(\begin{array}{ccc}
Z^{\prime} & 0 & 0 \\
0 & Z^{\prime \prime} & 0 \\
0 & 0 & z
\end{array}\right), \\
& {[\Pi, K]=\left(\begin{array}{ccc}
\Pi^{\prime} & 0 & K^{\prime} \\
0 & \Pi^{\prime \prime} & K^{\prime \prime}
\end{array}\right),}
\end{aligned}
$$

respectively; $K$ is the directed-tieline-on-node incidence matrix, the rows are for the nodes and the columns are for the tielines. I call the particularization (2) a reduction.

\footnotetext{
* Received January 5, 1979; revised version received May 6, 1980.

${ }^{1}$ By a dangling cutset, I mean a set of lines in a non-separable network which when cut leave the network connected but separable. A system of isolated networks interconnected by tielines in each cable of which there is a grounding wire is irreducible.
} 


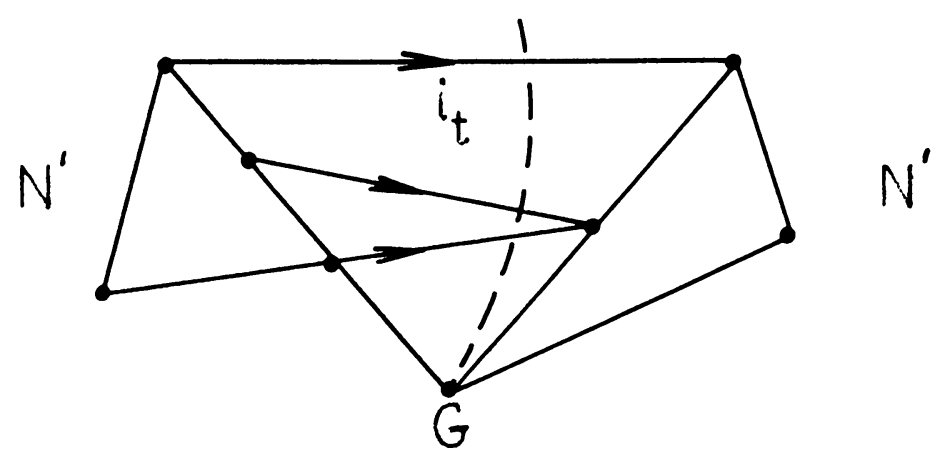

FIG. 1.

The e.m.f. equation for $n$ co-grounded inductively isolated networks interconnected in general by $n(n-1) / 2$ cables of tielines is of the form

$$
\left(\begin{array}{ccc}
Z & 0 & \Pi_{T} \\
0 & z & K_{T} \\
\Pi & K & 0
\end{array}\right)\left(\begin{array}{c}
i \\
i_{t} \\
v
\end{array}\right)=\left(\begin{array}{c}
0 \\
e_{t} \\
0
\end{array}\right)
$$

in which $Z$ is in $n$ diagonal blocks [1, Eq. (8), p. 217]. It is assumed that each of the $n$ networks has the same grounding point or is connected to ground by one of a radial system of grounding wires and that then $Z$ is in $n$ diagonal blocks of the form $\left[Z_{N_{i}}, r_{i}\right)$ in which $Z_{N_{i}}$ is the impedance matrix for the branches of the $i$ th network and $r_{i}$ is the impedance of a wire connecting the grounding-point of $N_{i}$ to the common ground-point $G$. As part of the solution of the problem (3) it was found that

$$
v=\mathscr{Z} \Pi Y e+\mathscr{Z} K y e_{t}
$$

where $\mathscr{Z}=\mathscr{Y}^{-1}$,

$$
y=\left(\Pi Y \Pi_{T}+K y K_{T}\right)
$$

in which, by definition and by virtue of the order of indexing the branches and nodes from network to network in parallel and then indexing the tielines from cable to cable, we get $\Pi Y \Pi_{T}$ in diagonal block form and so $\mathscr{Y}$ then comparatively easy to invert by the Bückner formula ${ }^{2}$ getting [1, p. 218]

$$
\mathscr{Z}=Z-Z K_{y} K_{T} Z=Z\left[Y-K_{y} K_{T}\right] Z
$$

where, by definition,

$$
\begin{aligned}
& Y=\left(\Pi Y \Pi_{T}\right), \\
& Z=Y^{-1}, \\
& y=\left[z+K_{T} Z K\right]^{-1} .
\end{aligned}
$$

\footnotetext{
${ }^{2}$ The formula was first discovered by Woodbury. Proofs of the formula were given by Braae, Synge and Bueckner. See Hans Edelmann, Berechnung elektrischer Verbundnetze, Springer, 1963, p. 232, for another proof and related history.
} 
With $Y$ and $z$ being diagonal or in diagonal blocks, it is easier to make these calculations and so get $v$ by (4) than to invert the matric coefficient in (3) when the branches and nodes are indexed in random order. Knowing $v$, we have that $i$ and $i_{t}$ are best computed from the equations

$$
i=Y\left(e-\Pi_{T} v\right), \quad i_{t}=y\left(e_{t}-K_{T} v\right)
$$

from the first two lines of (3).

From (4) we have the equation

$$
\mathscr{Y} v=\imath^{*}+K y e_{t}
$$

in which we write $\imath^{*}$ for $\Pi T e$ and have a vector of as many components as there are nodes in the complex and so, perhaps, thought of as a vector of currents entering the complex from the outside, as when the complex is a subnetwork of some larger network.

Consider now a shift of terms associated with the vector $l^{*}$ :

$$
\mathscr{Y} v=\imath^{*}+K y e_{t} \rightarrow Y v=\imath^{*}+K i_{t} .
$$

The result of this shift in combination with the equation

$$
K_{T} v+z i_{t}=e_{t}
$$

from the second row of (3) may be written as the matrix problem

$$
\left(\begin{array}{rr}
\boldsymbol{Y} & -\boldsymbol{K} \\
\boldsymbol{K}_{T} & z
\end{array}\right)\left(\begin{array}{l}
v \\
i_{t}
\end{array}\right)=\left(\begin{array}{l}
\imath^{*} \\
e_{t}
\end{array}\right)
$$

and the solution of this problem is, by inversion of the matric coefficient,

$$
\left(\begin{array}{l}
v \\
i_{t}
\end{array}\right)=\left(\begin{array}{cc}
\mathscr{Z} & Z K_{y} \\
-y K_{T} Z & y
\end{array}\right)\left(\begin{array}{l}
\imath^{*} \\
e_{t}
\end{array}\right) .
$$

This is a solution of (3) for $i_{t}$ and $v$ in terms of $\Pi Y e$ and $e_{t}$.

In this age of the electronic computer, an algebraic solution may of less value than simplicity in the formulation of the problem.

The last equation on p. 217 of [1] says

$$
i_{t}=-y K_{T} \mathscr{Z} \Pi Y e+\left[y-y K_{T} \mathscr{Z} K y\right] e_{t}
$$

and so, when $e=0$, and as can be verified by substitution,

$$
e_{t}=\left[z+K_{T}\left(\Pi Y \Pi_{T}\right)^{-1} K\right] i_{t} .
$$

This equation shows that given a set of currents $i_{t}$ entering the nodes of $N^{\prime \prime}$ of Fig. 1, say, we can specify the tieline e.m.f.'s necessary and sufficient to produce this current whatever the complexity of $N^{\prime}$. That is, given any passive connected network $N$ whatever, with branch-admittance matrix $Y$, and given any vector of currents $l$ forced in at such nodes as are specified by an incidence matrix $K$, the equation

$$
\left(\Pi Y \Pi_{T}\right) v=K_{l}
$$

can be realized only by an outside set of active dipoles either directly grounded or grounded through some other passive network. It cannot be realized otherwise: there are no e.m.f.s anywhere else which will produce a given set of currents directed over passive 
tielines to such nodes of $N$ as are specified by an incidence matrix $K$ : Eq. (16) implies that $v$ is a function of $l$ if $l$ exists. If we cannot specify what e.m.f.s produce the currents $l$ we cannot claim the currents exist.

If we premultiply both sides of (12) by the matrix $\left[1,0 / 0, \Gamma_{T}\right]$ and substitute $\Gamma i_{t}^{\prime}$ for $i_{t}$ we get an equation

$$
\left(\begin{array}{ll}
\Pi Y \Pi_{T} & -K \Gamma \\
\Gamma_{T} K_{T} & \Gamma_{T} z \Gamma
\end{array}\right)\left(\begin{array}{l}
v \\
i_{t}^{\prime}
\end{array}\right)=\left(\begin{array}{c}
i^{*} \\
e_{t}^{\prime}
\end{array}\right)
$$

which seems to be what Brameller, John and Scott [3, p. 221] have in mind as their extended and final form of the diakoptic problem as understood. But we have only to recognize the tielines as chords from the tree of one network to the tree of another network of the system to see that $\Gamma$ here can be only the identity matrix or a permutation by rows or columns of an identity matrix. Thus the matrix $\left[1,0 / 0, \Gamma_{T}\right]$ has an inverse $[1,0 / 0, \Gamma]$ and the solution of (17), to wit

$$
\left(\begin{array}{l}
v \\
i_{t}^{\prime}
\end{array}\right)=\left(\begin{array}{cc}
\mathscr{Z} & Z K_{y} \Gamma \\
-\Gamma_{T} K_{T} Z & \Gamma_{T} y \Gamma
\end{array}\right)\left(\begin{array}{l}
i^{*} \\
e_{t}^{\prime}
\end{array}\right),
$$

is similar to the solution of (12) just as the corresponding problems are similar, i.e., similar by a similarity transformation.

To sum up, the solution of the general diakoptic problem (3) was given in [1]. When the numerical values of the currents in the branches of the networks of the system are not of immediate interest, the subproblem (10) has numerical computational advantage over (3). Also, the algebraic solution $v=Z K_{y} e_{t}$ from (13) in the case of passive networks is simpler to work out numerically than is the solution $v=\mathscr{Z} K y e_{t}$ from (4) when all e.m.f.s other than those in the tielines are zero. When they are not all zero and $v=v\left(e, e_{t}\right)$, then the solution (13) for $v$ and the solution (4) have to be compared and it is seen that there is no advantage in the shift and even a small disadvantage. The solution for $v$ in (18), where the components of $i_{t}^{\prime}$ and $e_{t}^{\prime}$ are merely permutations of the components of $i_{t}$ and $e_{t}$ and not circuit values, is even less advantageous.

\section{REFERENCES}

[1] W. H. Ingram, On the inversion of the Cauer-Routh matrix, Quart. Appl. Math. 27, 215-222 (1969)

[2] W. H. Ingram, On incidence matrices implied in Maxwell's network theory, Quart. Appl. Math. 31, 137-141 (1973); Cauer's diophantine problem was $\Pi \gamma=0$ and Eq. (6) should read $i^{\prime}=\Pi^{\prime} i$. The equations on p. 220 of [1] are all correct and so the footnote on p. 140 of [2] which says that one of them is not correct, is false.

[3] Brameller, John and Scott, Practical diakoptics for electrical engineers, Chapman \& Hall, 1969. Taking into account their pre-1969 source-list of papers and books, pp. 239-240, their production, on p. 143, of Eq. (12) and later of (17) is most notable. 